\title{
Spectroscopic and photometric monitoring of southern post-AGB stars
}

\author{
D.J. Pooley, P.L. Cottrell, K.R. Pollard, M.D. Albrow \\ Mt John University Observatory, Department of Physics $\mathcal{G}$ Astronomy, \\ University of Canterbury, Private Bag 4800, Christchurch, New Zealand
}

\begin{abstract}
We present the results of contemporaneous photometric and spectroscopic monitoring of 20 post-AGB stars from Mt John University Observatory. Photometric measures were carried out using Johnson $B V$ and Cousins $R I$ filters, and the radial velocity measurements were acquired using spectra from an echelle spectrograph. Our programme spanned five years and the stars covered a range of spectral types from $\mathrm{B}$ to $\mathrm{K}$ in order to investigate the behaviour of post-AGB stars as they evolve away from the AGB. A number of stars proved to be variable in ways incompatible with post-AGB models and are reclassified. Periodicities are presented for a number of stars. Photometrically, HD 70379 was found to be pulsating in two modes with periods of 85 and $97 \mathrm{~d}$. The radial velocities also varied, with the peak amplitude occurring when the photometry was also changing most. AI CMi presented three different types of spectra associated with photometric brightness, with varying strengths of narrow emission lines and molecular bandheads. The $\mathrm{H} \alpha$ profiles in almost all of the stars show evidence of emission which varies on time scales of days to months. The $\mathrm{NaD}$ line profiles are generally complex showing between 4 and 7 components due to both circumstellar and interstellar material.
\end{abstract}

\section{Introduction}

The post-AGB phase is a very short transitional stage in the life of low to intermediate mass stars. Such stars will evolve rapidly from a cool AGB object to a hot white dwarf in only a few tens to a hundred thousand years. Many candidate post-AGB stars have been listed based upon a supergiant spectrum combined with an infrared excess (e.g. Trams et al. 1993; Oudmaijer et al. 1992). It is from these lists that we have drawn our sample.

Contemporaneous $B V R I$ photometry and high-resolution $(R \sim 30000)$ optical spectroscopy were acquired from Mt John University Observatory (MJUO) over a five year period starting in 1997 and extending into 2002.

\section{Photometry}

Most of the stars in our programme are variable. An average star would have a semi-regular variation in $V$ of about $0.2 \mathrm{mag}$ (peak-to-peak) with a quasi- 


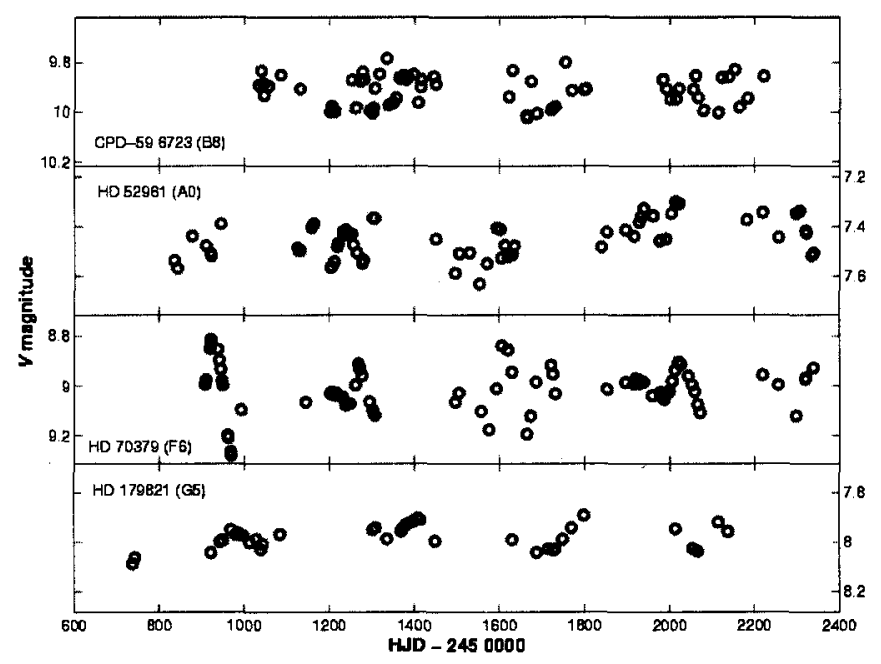

Figure 1. MJUO $V$-band photometry for four post-AGB stars. The stars cover a range of spectral types and therefore different stages in their post-AGB evolution.

period of about $100 \mathrm{~d}$. In general, the cooler stars had longer periods and larger amplitudes. Several stars showed almost no variation, i.e. less than 0.06 mag. HD 53300 is the least active of the stars showing no variation in radial velocity or in $\mathrm{H} \alpha$ line profile (which has no emission component).

The largest amplitude variation (1.5 mag) is that of the RV Tauri-like star AI CMi. Comparison with earlier photometry (Eggen 1983) suggests that there has been redistribution of power in the different modes of oscillation.

HD 70379 (see Fig. 1) showed a sinusoidal variation which has vanished in our second season. However, it returned only to fade a second time in 2000 (HJD 2451820 ). A closer analysis revealed two main periods at 85 and $97 \mathrm{~d}$ which interact and beat with a period of about $700 \mathrm{~d}$.

HD 95767 was a highly unusual object. Our early observations found that it had a period of $104 \mathrm{~d}$ which, like HD 70379, faded in the next season but was replaced with a general depression in the light level at HJD 2451600 . Around this time the spectra also radically changed, with emission components emerging in many low excitation potential lines, and $4 \AA$ of additional emission in $\mathrm{H} \alpha$.

\section{Radial Velocities}

Binarity has been suspected of playing a significant role in the evolution of postAGB stars and evidence of this, as well as any radial pulsations, were searched for in the spectroscopic data (see Fig. 2). HD 52961 ( $P \sim 1200 \mathrm{~d}$ ), HD 95767 $(P>1500 \mathrm{~d})$ and HD $108015(P \sim 871 \mathrm{~d})$ showed velocity changes consistent with being binaries. The typical radial velocity amplitude observed for the stars was $10 \mathrm{~km} \mathrm{~s}^{-1}$. The B-stars in our sample have velocity gradients consistent with an expanding atmosphere. 


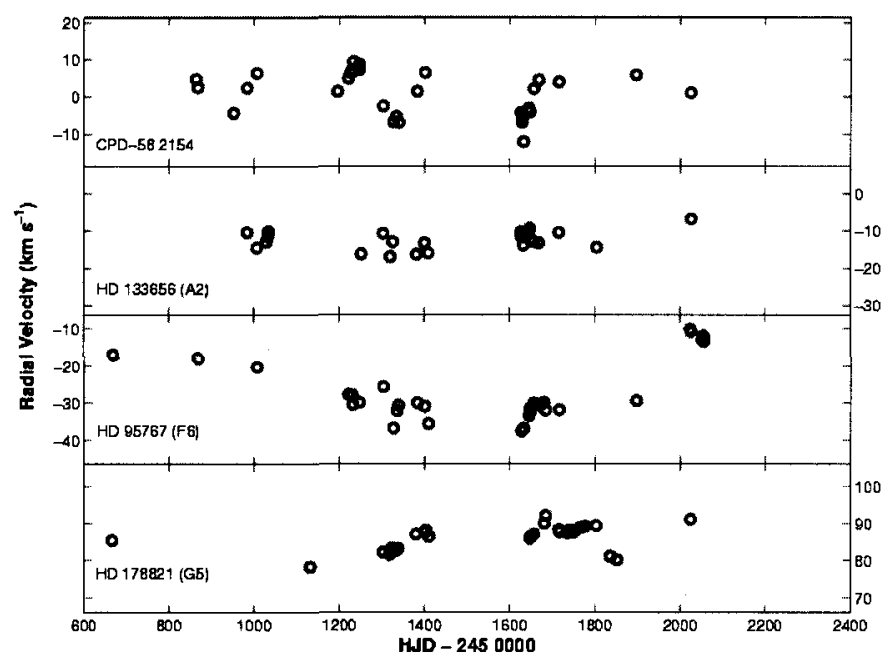

Figure 2. MJUO radial velocities for four post-AGB stars. The overall variations resemble those of the photometry. HD 95767 is a binary, showing long-term modulation of its radial velocity.

A number of stars showed mean radial velocities which were inconsistent with motion within the galactic disk. Most anomalous was HD $172481\left(V_{r} \sim\right.$ $-90 \mathrm{~km} \mathrm{~s}^{-1}$ ) which is probably in the Galactic halo. Only HD 70379 showed clear evidence of a link between the photometry and changes in the radius, with larger amplitudes being observed during the times of larger photometric activity. There is also a reduction in the strength of neutral metal lines when the star is brighter, consistent with an increase in the surface temperature when the star is smaller. During these times the star changes in size by about $30 \mathrm{R}_{\odot}$.

\section{4. $\mathrm{H} \alpha$ and $\mathrm{NaD}$}

There is a common thread to these stars in that their $\mathrm{H} \alpha$ profiles are essentially just subtly-modified photospheric lines. The dominant component is the wide absorption of the star, which is accompanied by two small emission features adjacent to the deep central part of the absorption (see Fig. 3). These are subject to changes in intensity on timescales of weeks to months. They are, on the whole, highly reminiscent of $\mathrm{H} \alpha$ profiles seen in globular cluster red giants (Cohen 1976; Smith \& Dupree 1988) with similar timescales, and nature, of the changes. The only difference appears to be increased absorption due to the earlier spectral types of our stars. From Cohen's simple recombination model it is possible to estimate the rate of mass loss as being of the order of $10^{-7} \mathrm{M}_{\odot} \mathrm{yr}^{-1}$. This agrees well with the estimates from models of mass-loss during this phase (e.g. Schönberner \& Steffen 2001). For the hottest stars the $\mathrm{H} \alpha$ line showed a strong P-Cygni profile. In the case of HD 168625, this was extremely variable and combined with a number of other emission components. 

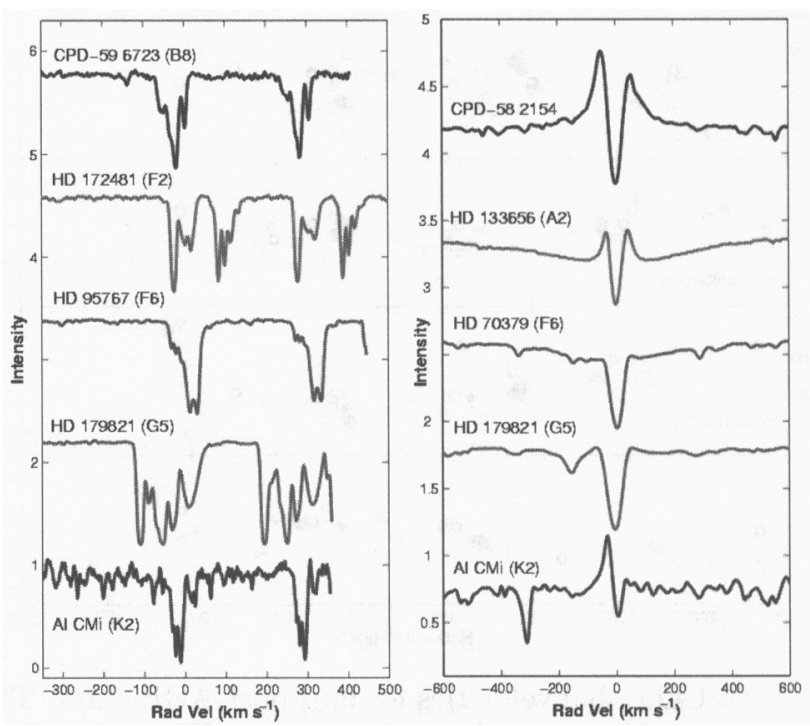

Figure 3. $\quad \mathrm{NaD}$ (left) and $\mathrm{H} \alpha$ (right) line profiles for selected postAGB stars shown in the respective stellar rest frames. The NaD profiles are complex and lack a photospheric absorption feature. The $\mathrm{H} \alpha$ profiles are quite similar, with varying amounts of emission combined with a wider photospheric absorption feature.

The NaD line profiles are very complex, containing up to 7 components. In a few cases it was possible to detect interstellar components using other field stars, or from the shape and radial velocity linking them with the Galactic spiral arms. A number of circumstellar components were also found, but only a few of the line profiles clearly exhibited stellar absorption.

Acknowledgments. The authors wish to thank Alan Gilmore and Pam Kilmartin for collecting the photometric data used in this paper.

\section{References}

Cohen, J.G. 1976, ApJ, 203, L127

Eggen, O.J. 1983, AJ, 88, 386

Oudmaijer, R.D., van der Veen, W.E.C.J., Waters, L.B.F.M., Trams, N.R., Waelkens, C., Engelsman, E. 1992, A\&AS, 96, 625

Schönberner, D., Steffen, M. 2001, in Post-AGB objects as a phase of stellar evolution, ed. R. Szczerba \& S.K. Górny (Dordrecht: Kluwer), 85

Smith, G.H., Dupree, A.K. 1988, AJ, 95, 1547

Trams, N.R., Waters, L.B.F.M., Waelkens, C. 1991, A\&AS, 87, 361 